\title{
Community diversity and succession in fermented grains during the stacking fermentation of Chinese moutai-flavored liquor making
}

\author{
Liang $\mathrm{ZHAO}^{1}{ }^{(\mathbb{D})}$, Xinliang $\mathrm{MO}^{1}$, Chunlin $\mathrm{ZHANG}^{1}$, Liang $\mathrm{YANG}^{1}$, Xinye WANG ${ }^{1 *}$
}

\begin{abstract}
As an indispensable and crucial part of the Moutai-flavored liquor production process, stacking fermentation facilitates the accumulation of various microbiota as microbial materials in fermented grains used for liquor making. The diversity of and dynamic changes in highly complex microbiota during this fermentation phase were investigated by characterizing microbial communities through high-throughput sequencing with an Illumina PE300 platform. Through community composition and differentially abundant analyses, 12 key bacteria and 12 key fungi were identified, which contributed to major variations in community succession. Bacillus and Saccharomyces were overrepresented in the bacterial and fungal communities, respectively. In addition, differentially abundant analyses showed that the key microbiota were mainly structured from the early period to the middle period of fermentation and functional populations showed a large accumulation in later periods. Further, endogenous factors showed changing trends, and their potential correlations and varied interactions with the key microbiota were explored through PLS-DA modeling. All the findings in the current study enhances knowledge of complex key microbiota in stacking fermentation and provides theoretical support for optimizing microbial structures through the regulation of endogenous factors and improving fermentative quality.
\end{abstract}

Keywords: microbiota; community diversity; endogenous factors; differentially abundant taxa.

Practical Application: Deep understanding of the microbial succession and its relationship to the endogenous factors is the basis of monitoring and optimizing the fermentative condition in Moutai-flavored liquor making and therefore, help to guarantee the quality and quantity of liquor.

\section{Introduction}

Similar to whisky in Scotland and brandy in France, Moutaiflavored liquor is a symbolic drink in China; (Wang et al., 2008; $\mathrm{Xu} \& \mathrm{Ji}$, 2012). Moutai-flavored liquor is popular because of its production method, quality, and complex aromas (Wu et al., 2016). The entire production process of this liquor includes Daqu making, stacking fermentation, anaerobic fermentation in pits, and distillation (Xiong, 1994). Daqu can be used as a starter for liquor fermentation and next batch for Daqu making. During stacking fermentation, distilled fermented grains (FGs) are mixed with powdered mature Daqu. The grain mixture is then piled up in a cone-shaped stack on the ground for 3-11 days, depending on the temperature of the environment. The process is terminated when the temperature on the top of the stack reaches $45-50{ }^{\circ} \mathrm{C}$. This stage is crucial to the structuring and accumulation of key microbiota, thus providing microbial materials for subsequent anaerobic fermentation. In anaerobic fermentation, FGs from stacking fermentation are placed into underground cubic pits and sealed for approximately 30 days of anaerobic fermentation. This process is mainly used for the production of alcohol, as well as aromatic substances. The FGs are then distilled for the collection of raw liquor (Wang et al., 2018).

Most studies on the Moutai-flavored-liquor-making process are focused on Daqu making and the anaerobic fermentation phase (Wang et al., 2008; Yang et al., 2011, 2019; Xiu et al., 2012; Wu et al., 2013, 2015; Song et al., 2017) because the quality and quantity of liquor production are directly attributable to this process. However, diverse microbiota utilized in pit fermentation accumulate in FGs during stacking fermentation. In other words, the quality of stacking fermentation determines that of pit fermentation. Given that shifts in microbial communities during stacking fermentation and the mechanisms and factors promoting microbial succession are already known, we may adopt measures that can shift the direction of microbial development to our expectations and build a good basis for anaerobic fermentation in pits.

Chinese Moutai-flavored liquor making is based on multiple fermentation batches; that is, eight fermentation rounds mainly consisting the entire production cycle that lasts for a year (Yang et al., 2019). Seven raw liquors from the second round to the eighth round differ in quality and flavor presumably because of diverse microbiota, which vary according to air temperature and climate during the production rounds. Raw liquor from the third fermentation round exhibits high quality and excellent flavor. We focused on this fermentation round and aimed to determine dynamic changes in microbial diversity during the stacking fermentation process through the next-generation 
sequencing of bacterial V3 and V4 and fungal ITS2 of rRNA genes with Illumina MiSeq. Microbial succession is inevitably influenced by endogenous factors (e.g., temperature, lactic acid, moisture, and ethanol) in the fermentation process (Song et al., 2017). Therefore, the second aim of our study was to reveal how the factors are associated with community turnover. In addition, we further constructed a correlated network among microbial taxa in the course of fermentation to understand the mechanism underlying the microbial shift and uncover the functionality of stacking fermentation on microbial selection and accumulation.

\section{Materials and methods}

\subsection{Sample collection}

Samples were collected from Hongtudi distillery factory in Moutai town, Kweichow Province, China. The fermentation round targeted includes three distinct phases: stacking fermentation, anaerobic fermentation, and distillation. Samples were taken on the first (P1), third (P2), fifth (P3), seventh (P4), and eighth (P5) day after piling the FGs into a cone-shaped stack during the stacking fermentation phase. Samples were collected from five different locations in the stack (detailed information available in Figure S1) with a coring tool. The temperatures of the sampling locations were measured and recorded before sample collection. Finally, all the samples (five replicates for each time point) were transported to the laboratory and stored at $-80^{\circ} \mathrm{C}$.

\section{Chemical analysis of the FG samples}

Briefly, $10 \mathrm{~g}$ of FGs was mixed with $90 \mathrm{~mL}$ of distilled water, ultrasonically treated at $0^{\circ} \mathrm{C}$ for $30 \mathrm{~min}$, and centrifuged at $4{ }^{\circ} \mathrm{C}$ for $5 \mathrm{~min}$. The obtained supernatant was used in determining ethanol content through gas chromatography-mass spectrometry according to the methods of Wu et al. (2015). We also conducted reversed-phase high-performance liquid chromatography analysis to determine lactate acid content in the supernatant with the method of Bai et al. (2000). Moisture content in $10 \mathrm{~g}$ of samples dried at $135{ }^{\circ} \mathrm{C}$ for $1 \mathrm{~h}$ was determined through a gravimetric method immediately after sampling.

\subsection{DNA extraction and MiSeq sequencing}

Community DNA was extracted from $0.5 \mathrm{~g}$ of FG samples with an MP FastDNA ${ }^{\circ}$ SPIN kit for soil (MP Biochemicals, Solon, OH, USA) according to the manufacturer's protocol. The primer set (319F 5'-ACTCCTACGGGAGGCAGCAG-3'; 806R 5'-GGACTACHVGGGTWTCTAAT-3') was used to amplify the V3 and V4 regions of the 16S rRNA genes (Fadrosh et al., 2014). The fungal community was analyzed by targeting the ITS2 regions of the rRNA genes, and the primers fITS7 (5'-GTGARTCATCGAATCTTTG-3') and ITS4 (5'-TCCTCCGCTTATTGATATGC-3') were used to amplify these regions (Karlsson et al., 2014). Polymerase chain reaction (PCR) was conducted using $25 \mu \mathrm{L}$ (total volume) of mixture containing $25 \mathrm{ng}$ of genomic DNA extract, $0.625 \mathrm{U}$ of Ex Taq polymerase (Takara, Shanghai, China), $1 \times$ PCR buffer, $800 \mu \mathrm{M}$ of dNTP, and $300 \mathrm{nM}$ primer. The reactions were performed using a MasterCycler gradient thermal cycler (Eppendorf,
Hamburg, Germany) under the following cycling conditions: initial denaturation at $98^{\circ} \mathrm{C}$ for $30 \mathrm{~s} ; 35$ cycles of denaturation at $98^{\circ} \mathrm{C}$ for $10 \mathrm{~s}$, annealing at $54^{\circ} \mathrm{C}$ for $30 \mathrm{~s}$, and extension at $72{ }^{\circ} \mathrm{C}$ for $45 \mathrm{~s}$; and final extension at $72{ }^{\circ} \mathrm{C}$ for $10 \mathrm{~min}$.

PCR amplification was performed in triplicate. The products were pooled for each subsample. A composite DNA sample for sequencing was prepared by combining the equimolar ratios of the amplified products from the individual subsamples as described previously (Fierer et al., 2008). Composite DNA was gel-purified and sequenced using an Illumina MiSeq PE300 platform (Caporaso et al., 2012) at LC-Bio Co., Ltd., Hangzhou, ZheJiang, China.

\subsection{Sequence analysis}

Raw Illumina fastq files were de-multiplexed, quality filtered, and analyzed using QIIME v1.7.0 (Caporaso et al., 2010). Reads containing more than three consecutive bases and with a quality score of $<20$ or reads containing $>6 \mathrm{nt}$ of ambiguous nucleotides ("N") in their homopolymeric regions were discarded. Chimeric sequences were identified and removed using USEARCH (Edgar et al., 2011). Operational taxonomic units (OTUs) were assigned using UCLUST at a threshold level of $97 \%$ similarity (Edgar, 2010). Low-abundance OTUs with a total of at least five counts were eliminated from the OTU table. The representative sequences for each OTU were assigned to taxonomic groups with an RDP Classifier (Caporaso et al., 2011). The alpha microbial biodiversity (Shannon index and Chaol richness; Pagaling et al., 2014) of each sample was estimated using QIIME v1.7.0.

\subsection{Statistical analysis}

All statistical analyses were performed in the R environment (http://www.r-project.org) by using various packages. Endogenous factors and community diversities were compared among fermentation periods by performing Wilcoxon test using 'stats' package. Similarity analysis (Anosim; Clarke, 1993) and permutational multivariate analysis of variance (Adonis; Anderson, 2001) were conducted with 9999 permutations by using 'the vegan' package, which was used in determining whether the sample classifications (different fermentation periods) show significant differences in phylogenetic and compositional diversity according to weighted UniFrac distances and Bray-Curtis dissimilarity matrices, respectively. Differentially abundant genera were detected using the EdgeR (Robinson et al., 2010; McMurdie \& Holmes 2014) generalized linear model approach. Correlations among microbiota and endogenous factors were determined using a PLS-DA model and plotted in a concatenation panel using the mixOmics package (Rohart et al., 2017).

\section{Results}

\subsection{Taxonomic distributions of bacteria and fungi}

After quality filtering and the removal of chimeric sequences and low-abundance OTUs, we obtained 1,140,782 exploitable sequences ( $90.0 \%$ of total sequences) from all the FG samples. The sequences were classified in 7,929 OTUs, with an average of 
1111 per sample $(\max =2335, \min =65, \mathrm{sd}=584)$. The predominant taxa in the bacterial community included those belonging to the orders Bacillales (relative abundance $88.9 \%$ ) and Lactobacillales (6.7\%). The main genera were Bacillus (46.9\%), Lentibacillus (15.4\%), Thermoactinomyces (11.5\%), Lactobacillus (6.0\%), Scopulibacillus (5.6\%), and Kroppenstedtia (5.0\%). Other genera, including Oceanobacillus (4.2\%) and Erwinia (1.1\%), were also observed and had minor relative abundances (Figure 1).

By contrast, we obtained 2,153,696 exploitable sequences (99.3\% of total sequences) from fungal communities across FG samples, which can be assigned to 3,351 OTUs, with a mean of 406 per sample $(\max =799, \min =122$, $s d=213)$. Taxonomic analysis showed that the fungal community was overrepresented by yeast (62.7\%), particularly Saccharomyces (51.2\%), Zygosaccharomyces (6.6\%), Saccharomycopsis (2.7\%), and Pichia (1.2\%). The community contained notable filamentous fungi, such as Byssochlamys (9.0\%) and Monascus (7.7\%), and thermophilic fungi, such as genus Thermomyces (12.5\%), Thermoascus (4.5\%), and Rasamsonia (1.0\%). Moreover, other unclassified fungi had a relative abundance of 3.5\% (Figure 1). Saccharomyces cerevisiae, Zygosaccharomyces bailii, Saccharomycopsis selenospora, Pichia burtonii, Monascus purpureus, Byssochlamys spectabilis, Thermomyces lanuginosus, Thermoascus aurantiacus, and Rasamsonia composticola were exclusively or predominantly included in these fungal genera.

Overall, Firmicutes and Ascomycota were dominant at the phylum level, comprising approximately $95.8 \%$ and $98.8 \%$ of bacteria and fungi communities, respectively.

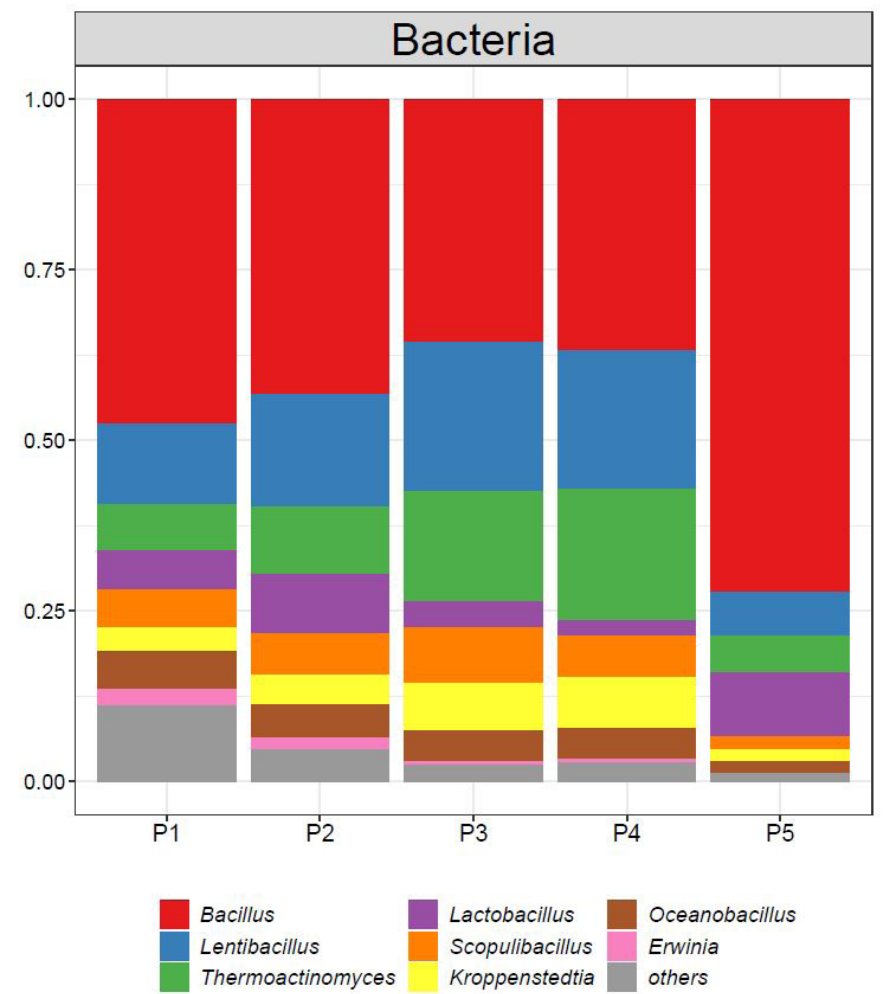

\subsection{Changes in endogenous factors and community diversities}

Dynamic changes in endogenous factors, including temperature, moisture, ethanol, and lactic acid (Table S1), were analyzed. Changes in these factors are displayed in Figure 2a. Ethanol and temperature significantly increased after 5 days (P3) and then reached their highest levels (ethanol: $0.70 \mathrm{~g} / 100 \mathrm{~g}$ FGs; temperature: $36.32{ }^{\circ} \mathrm{C}$ ) at P5. Lactic acid and moisture contents peaked at $\mathrm{P} 3$ and $\mathrm{P} 4$ (lactic acid: $1.76 \mathrm{~g} / 100 \mathrm{~g}$; moisture: 0.47), respectively, but declined at P5 (lactic acid: $1.48 \mathrm{~g} / 100 \mathrm{~g}$; moisture: 0.45 ).

Changes in bacterial and fungal diversity are shown in Figure 2b (also Table S1). In general, bacterial diversity had a declining trend, especially Chao 1 richness. The fungal diversity showed a fluctuant change across the entire fermentation process. For example, Chaol richness significantly increased, and three times that in P1 after 2 days of fermentation. However, it significantly decreased at P4 and P5. Shannon diversity showed a slight increase from P1 to P2 but significantly declined at P4 and P5.

\subsection{Succession patterns of the microbial communities}

The bacterial and fungal communities had significant differences in phylogenetic and compositional diversity at distinct periods, as demonstrated by Anosim and Adonis tests (Table 1), indicating significant succession in the course of fermentation. For the identification of the microbial taxa that significantly responded to the fermentation process, the abundances of the OTUs were combined on the genus level, and edgeR analyses

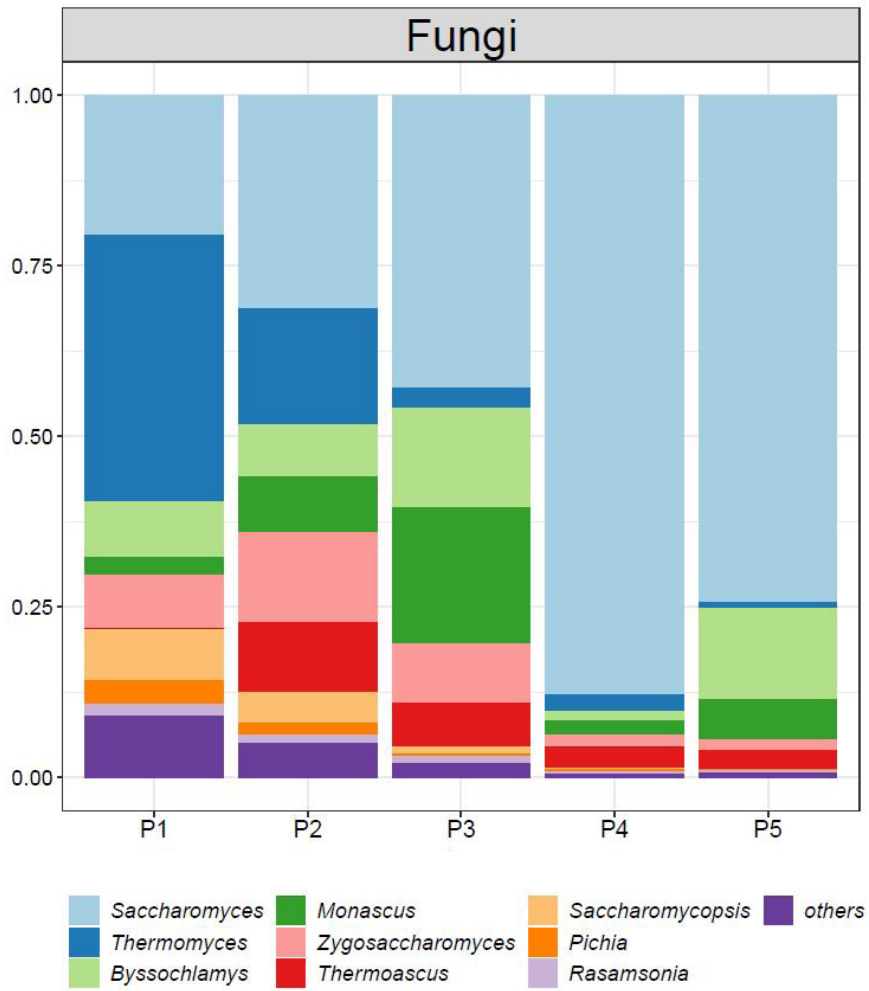

Figure 1. Relative abundance (\%) of dominant genera in bacterial and fungal communities of FGs at different fermentation periods. 

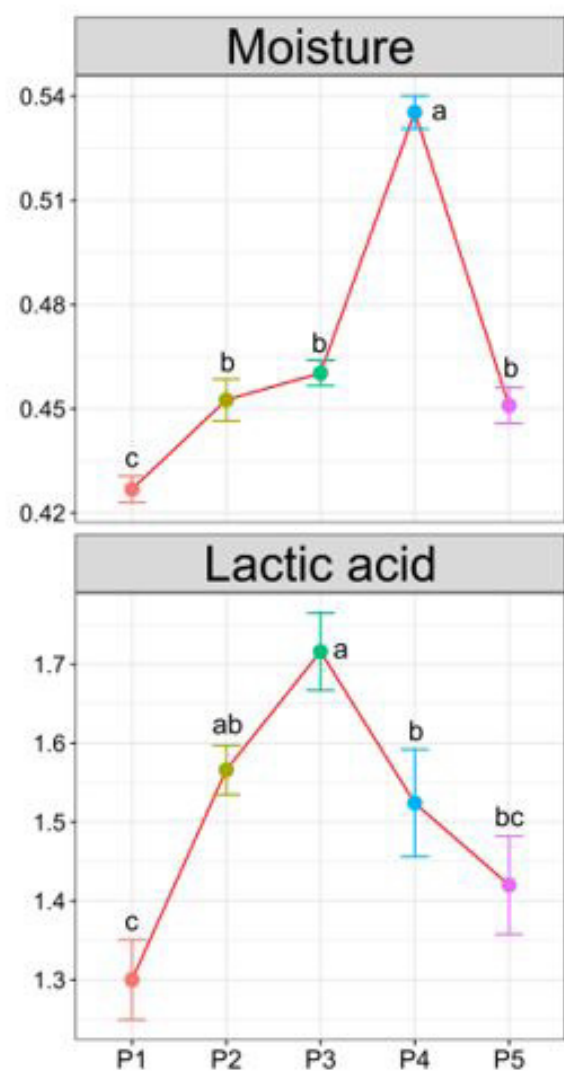

Shannon diversity

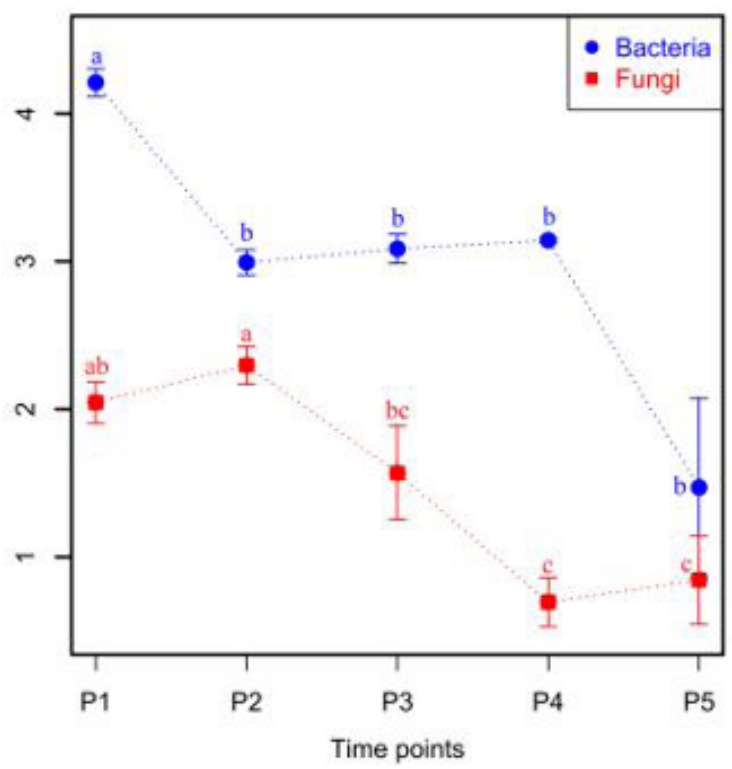

Figure 2. Changes in (a) endogenous factors and (b) community diversities of FGs during fermentation process. Error bars represent the standard error of the five sample replicates in each period.

were performed. Genus abundance in the initial period (P1) was used as the control with an adjusted $P$-value cutoff of 0.05 . The adjusted $P$-values for multiple comparisons were calculated

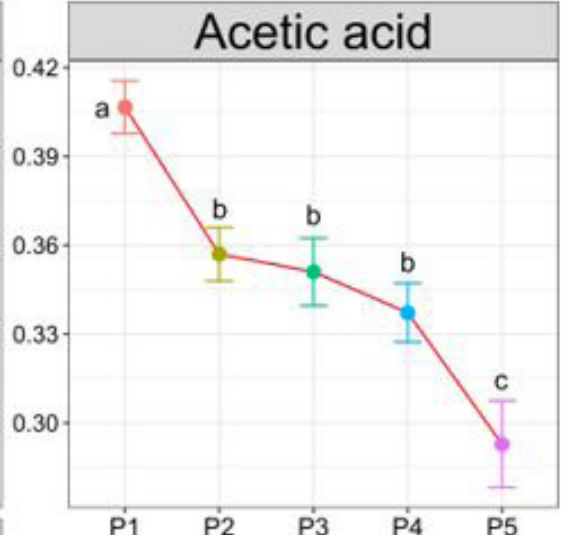

Chao1 richness

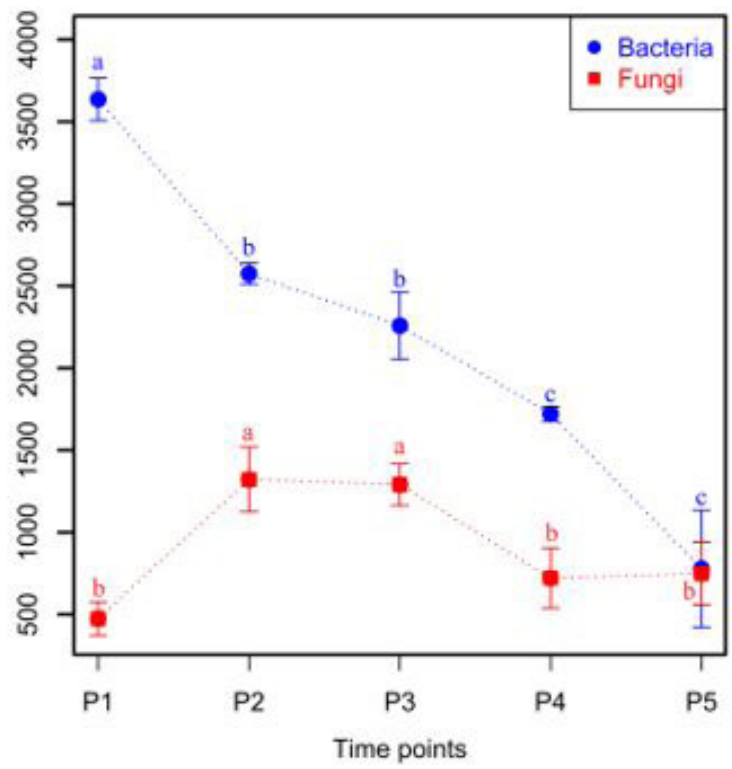

using Benjamini and Hochberg's algorithm (also known as false discovery rate). The results showed that 45 out of 411 bacterial genera and 15 out of 186 fungal genera significantly changed 
Table 1. Results of Anosim and Adonis tests on phylogenetic and compositional diversities in the communities of the two domains among different period.

\begin{tabular}{ccc}
\hline Domain & Phylogentic diversity (Weighted Unifrac distances) & Compositional diversity (Bray-Curtis dissimilarities) \\
\hline Bacteria & Anosim: $\mathrm{R}=0.50, P<0.001$ & Anosim: $\mathrm{R}=0.50, P<0.001$ \\
& Adonis: $\mathrm{R}^{2}=0.51, P<0.001$ & Adonis: $\mathrm{R}^{2}=0.53, P<0.001$ \\
Fungi & Anosim: $\mathrm{R}=0.25, P=0.0011$ & Anosim: $\mathrm{R}=0.45, P<0.001$ \\
& Adonis: $\mathrm{R}^{2}=0.37, P=0.0075$ & Adonis: $\mathrm{R}^{2}=0.56, P<0.001$ \\
\hline
\end{tabular}

during fermentation (detailed information available in Table S2), accounting for $51.8 \%$ and $78.0 \%$ of abundances in the bacterial and fungal communities, respectively. Three bacterial genera were enriched in P5, whreas 24, 25, 24, and 31 were depleted in P2, P3, P4, and P5, respectively. P2-P5 enriched one, five, two, and four fungal genera and depleted six, three, six, and five, respectively. These results indicated an assemblage effect for fungal microbes but an exclusive effect for bacterial microbes, as shown in Figure 3a. In the Venn diagram (Figure 3b), overlaps in differentially abundant genera were observed between time points. Of the 24 bacterial genera depleted in P2, 18, 16, and 18 were significantly depleted in P3, P4, and P5, indicating that most of the microbes depleted during fermentation may have been repressed at the early stage. In fungal communities, microbes were mainly enriched from $\mathrm{P} 2$ to $\mathrm{P} 3$ because the genera enriched in P2, P4, and P5 were also enriched in P3. As in the bacterial communities, P2 acted as a "filtration" time point for fungal communities, and depleted microbes during fermentation were largely repressed at this stage. This result was indicated by the depleted genera in P2 shared with P3, P4, and P5.

The substantial components (relative abundance $>0.1 \%$ ) of the significantly changed genera in the bacterial communities were Bacillus, Pediococcus, Erwinia, Enterobacter, Weissella, and Pseudomonas, and those in fungal communities were Saccharomyces, Byssochlamys, Monascus, Thermoascus, Saccharomycopsis, Schizosaccharomyces, Xeromyces, and Chaetothyriales. Given that these and the dominant genera might contribute the major variations in the structures of communities, their contributions to the total variation in the community structure were calculated based on Bray-Curtis dissimilarities. The results showed that in the bacterial community, the six genera and the other six dominant genera totally accounted for $95.2 \%$ of the variation in whole community dissimilarities. Meanwhile, eight genera and the other four dominant genera of the fungal community accounted for $98.9 \%$ of the total variation in the whole community structure. Thus, these 12 bacterial genera and 12 fungal genera were defined as key microbiota because of their major roles in community succession during fermentation.

\subsection{Correlations between endogenous factors and key microbiota of bacteria and fungi}

The relationships among the five endogenous factors, 12 key bacterial taxa, and 12 key fungal taxa during the five stages of FGs were explored using the PLS-DA algorithm and visualized using a circus plot. A correlation cutoff of 0.7 was used, and the association among the three data sets are depicted in Figure 4. Moisture was positively correlated with
Saccharomyces, Thermoactinomyces, and Kroppenstedtia and negatively correlated with Thermomyces. Ethanol was positively correlated with Saccharomyces and negatively correlated with Saccharomycopsis, Thermomyces, Erwinia, Enterobacter, Weissella, and Pseudomonas. Acetic acid was positively correlated with Saccharomycopsis, Xeromyces, Thermomyces, Pichia, Rasamsonia, Erwinia, Enterobacter, Pediococcus, Weissella, and Pseudomonas. Ttemperature was positively correlated with Saccharomyces and negatively correlated with Saccharomycopsis, Rasamsonia, Erwinia, Enterobacter, Pediococcus, Weissella, and Pseudomonas. Lactic acid were positively correlated with Schizosaccharomyces, Lentibacillus, Thermoactinomyces, Scopulibacillus, and Kroppenstedtia but negatively correlated with Bacillus.

As shown in the circus plot (Figure 4), correlations between the key bacteria and fungi were detected, and total of 23 pairwise associations between the two data sets were identified. For example, Saccharomyces was negatively correlated with Erwinia, Enterobacter, Weissella, and Pseudomonas. Saccharomycopsis was positively correlated with Erwinia, Enterobacter, Pediococcus, Weissella, and Pseudomonas. Schizosaccharomyces was negatively correlated with Bacillus and positively correlated with Lentibacillus and Scopulibacillus. Thermomyces and Pichia were positively correlated with Erwinia, Enterobacter, Pediococcus, Weissella, and Pseudomonas. Rasamsonia was positively correlated with Weissella.

\section{Discussion}

We found 17 dominant genera in the course of stacking fermentation (Figure 1). Eight of them belonged to the bacterial domain, and 9 to the fungal domain. Lv et al. (2019) reported that Pichia microbes overrepresent fungal communities, followed by Saccharomyces in the first (Xiasha) and second (Zaosha) rounds of stacking fermentation. Sun et al. (2019) investigated the sixth round of fermentation, and their results showed that Paecilomyces and Candida microbes are predominant in the stacking stage. By contrast, our findings showed the predominance of Saccharomyces in the fungal community, as further evidenced by the large variations in the key microbiota of fungi among the rounds of fermentation. The dominant bacterial genera found in our work were nearly similar to those found in previous studies (Wang et al., 2019), suggesting that the major bacterial microbes are stable in all the rounds of fermentation.

In general, the communities of the two domains showed the decline trends of $\alpha$-diversity as reflected by the decreased Shannon indices and Chaol richness (Figure 2b). After estimating Shannon indices in the fourth round of stacking fermentation, Cheng et al. (2014) showed that the middle period had the highest 

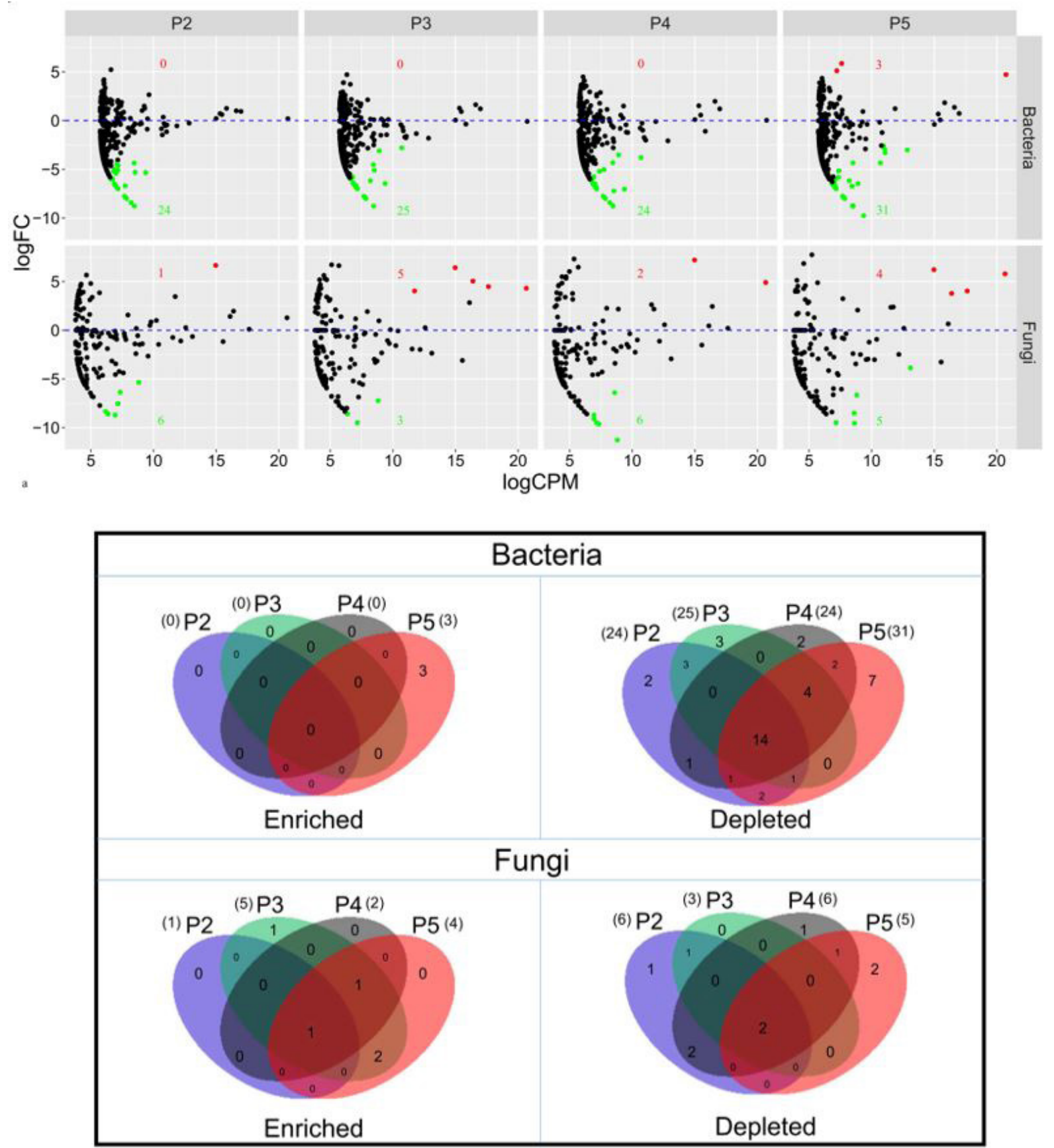

b

Figure 3. FGs enriched and depleted for certain microbial taxa (genus level) during the fermentation process. (a) Volcano-plot showing enrichment and depletion of the bacterial and fungal genera for each fermentative period compared with initial period (P1) as determined by differential abundance analysis. Each point represents an individual genus, and the position along the $y$ axis represents the abundance fold change compared with P1. The dots colored by red, green, or black indicated that genera were enriched, depleted, or changed without significance in certain periods compared with P1, respectively; (b) Numbers (in parentheses) of differentially enriched and depleted genera between each period compared with P1.

bacterial diversity, followed by the early and later periods. They discussed that the earlier period had the lowest diversity because of the low resolution of the DGGE method, which they used exhaustively to explore the microbes. Through high-throughput sequencing, a complementary and robust result was obtained in our study. Community compositions in FGs could become more simplification as compared with the earlier periods during fermentation.

In the present study, the clear filtration effect for bacterial microbes was observed in the fermentation process, that is, 


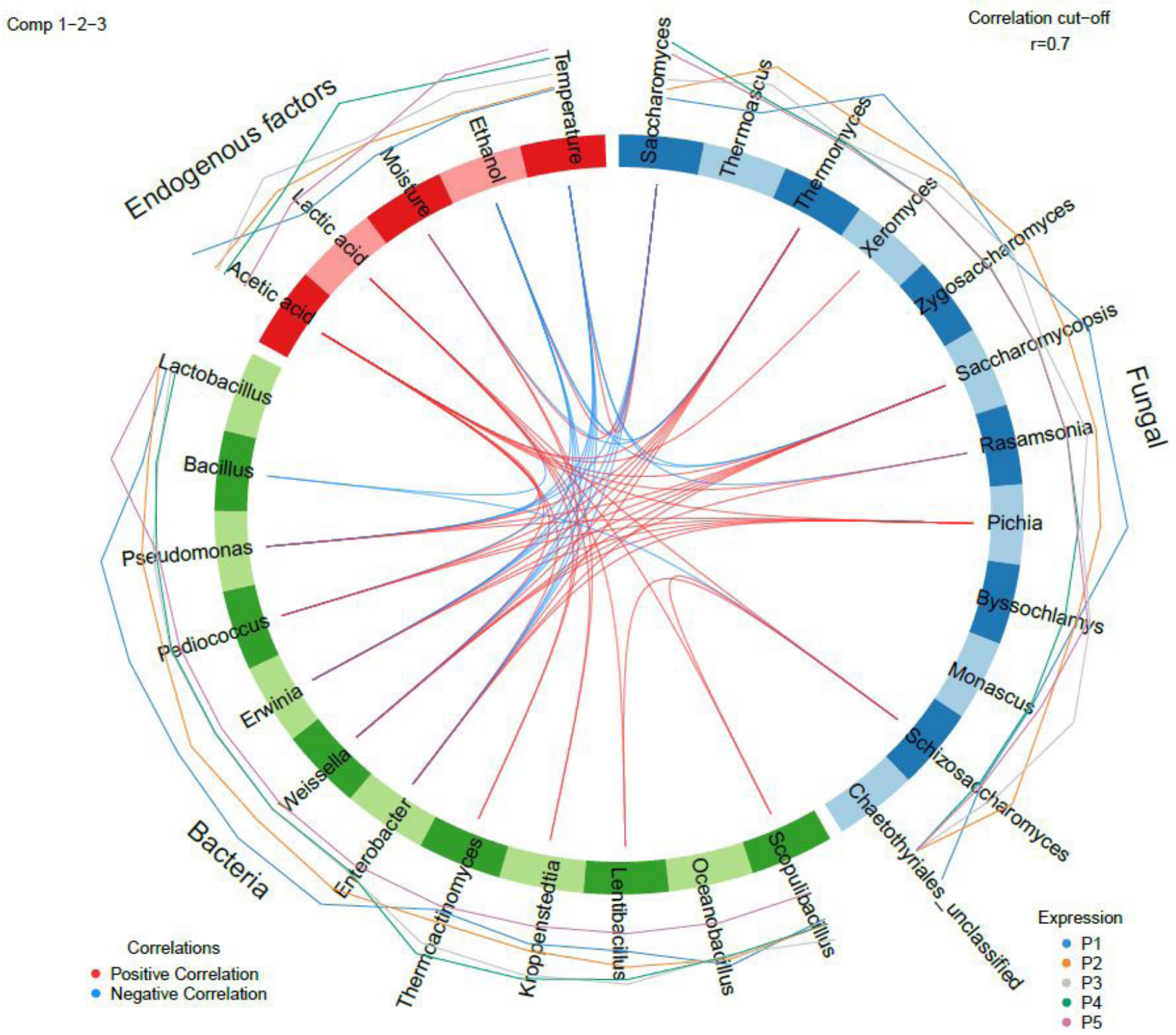

Figure 4. Correlation analysis between key microbiota and endogenous factors of FGs by PLS-DA modeling. Red lines in the circle represent positive correlation, whereas blue lines represent negative correlation between key microbiota and endogenous factors. Red blocks on the circle represent the five endogenous factors, blue blocks represent the 12 key bacterial genera and green blocks represent the 12 key fungal genera. Lines in different colors outside the circle represent the content of endogenous factors or the relative abundances of microbiota presented in each period, respectively.

42 out of the 45 significantly changed genera were depleted in the course of fermentation. By contrast, the fungal microbes were subjected to the effects of filtration and assemblage (Figure 3). These results indicated that fungal microbes are more capable of adapting to FG environment than bacterial microbes. The continuous increase in ethanol content may act as a repressing force for the survival of bacterial microbes in FGs. This finding may explain why major bacterial microorganisms seemed to be more stable than fungal microbes across different rounds of fermentation. In addition, Bacillus, which is one of the most important microorganisms in Moutai-flavored liquor production, was significantly enriched only in P5 (relative abundance $=72.1 \%$ ). Its relative abundance in the earlier periods ranged from $35.4 \%$ to $47.4 \%$. This result may be attributable to lactic acid, which had significantly higher levels in P2-P4 than in P1 and showed declined level at P5, in which lactic acid content was the same as that in $\mathrm{P} 1$. This condition promoted the growth of Bacillus microbes. The filtration effect of the FGs on the microbes was detected mainly at P2, and the FGs enriching functional microorganisms, such as Thermoascus, Monascus, Byssochlamys, Saccharomyces, and Schizosaccharomyces, were observed mainly at P3. These results suggested that the early and 
the middle stages were of great importance to the structuring of key composition for fermentation. In the later stage, from P4 to P5, Thermoascus, Monascus, Byssochlamys, and especially Bacillus and Saccharomyces, which are the most important microorganisms in Moutai-flavored liquor making (Wu et al., 2012, 2013; Song et al., 2017; Sun et al., 2019; Wang et al., 2018; Wang et al., 2019) accumulated, suggesting that the rapid growth of functional populations mainly occurred in the later stages.

The endogenous factors are important indicators for fermentative quality and strongly associated with microbial succession (Song et al., 2017). In our study, their potential correlations with key microbiota were revealed through PLS-DA modeling (Figure 4). Temperature was positive correlated with Saccharomyces only, showing that heat accumulation related to yeast growth is the reason for increase in FG temperature (Wu et al., 2013). Given that ethanol is mainly produced by yeast, its increase is indicative of the growth of Saccharomyces microbes, which was conveyed by the positively correlation only between ethanol and Saccharomyces. Moreover, the positive correlation of moisture with Saccharomyces, Thermoactinomyces, and Kroppenstedtia indicated the preferences of these microbes to relatively high water content. Adding an appropriate amount of water to the FGs in the stacking fermentation stage can increase liquor yields in the subsequent pit fermentation stage. This finding demonstrated the yeasts' preference for water, by which the yeast could be dramatically accumulated in the stacking and then be utilized for alcoholic production in pit. In addition, a high water content can promote the growth of Thermoactinomyces and Kroppenstedtia, which are dominant or core bacterial microorganisms in Moutai-flavored liquor fermentation (Ying et al., 2014; Huang et al., 2017).

The continuous accumulation of lactic acid gradually lowers $\mathrm{pH}$, thereby compromising the growth of microorganisms with poor acid resistance. Change in lactic acid concentration affects the balance of microbial consortium in FGs, affecting the enrichment of functional microbes in stacking fermentation, liquor quality, and yield. In an anaerobic fermentation system, Lactobacillus is a major lactate-producing microorganism, as revealed by its strongly positive correlation with lactic acid (Song et al., 2017; Wang et al., 2018). In facultative aerobic fermentation processes, such as stacking, lactic acid is produced not only by Lactobacillus but also by other communities. This hypothesis was supported by the results showing no correlation between Lactobacillus and lactic acid; moreover, the positive correlations of lactic acid with Schizosaccharomyces, Lentibacillus, Thermoactinomyces, Scopulibacillus, and Kroppenstedtia showed that microbes affiliated with these genera likely produced lactic acid. Although few studies directly indicated the contribution of these microbes to lactate production during liquor fermentation, especially in the stacking fermentation stage, all the genera except Scopulibacillus (no reference data) and Kroppenstedtia can convert pyruvate to lactate with their enzymes, as illustrated by KEGG database (Kanehisa et al., 2004). For example, these genera possess lactate dehydrogenase that can convert pyruvate to lactate by one step. Schizosaccharomyces can be used in converting malic acid to lactic acid in wine fermentation (Mylona et al., 2016) because it can synthesize malate dehydrogenase and lactate dehydrogenase, which convert malate to lactate via pyruvate. The enzymes used by
Kroppenstedtia in converting pyruvate to lactate are incomplete, and thus lactate cannot be synthetized by this genus theoretically. The positive correlation between this genus and lactic acid might be indirect because of the high correlations of this genus with Lentibacillus, Thermoactinomyces, and Scopulibacillus. These results provided insight into the major lactate-producing microbiota in facultative aerobic condition, but further studies are needed to investigate the metabolites and synergistic microbial effects of these genera. Bacillus species excrete extracellular products, such as proteases, lipase, thermostable $\alpha$-amylase, cellulase, and hemicellulase, which are useful in reactions leading to flavor production (Beaumont, 2002). Several flavor substances are integral parts of the flavor-producing composition of Moutai (Yang et al., 2011). Our results showed that Bacillus was negatively correlated with lactic acid, in general agreement with those of Wang et al. (2018). These findings suggested that the growth of Bacillus spp. can be repressed by lactic acid and the regulation of lactic acid content is important to enrichment of Bacillus microbes in FGs.

No typical acetic acid-producing bacteria were found dominant in this study. Other studies showed the same finding. Pediococcus and Weissella covered varied heterofermentative species, which were supposed to be the main contributors for acetic acid production, as reflected by the positive correlations among them. In addition, Song et al. (2017) suggested that Pichia, Saccharomyces, and Zygosaccharomyces can convert lactic acid to acetic acid via pyruvate and acetyl-CoA. Therefore, we extrapolated that acetic acid might be derived from the above microbiota. As to the other members that had positive correlations with acetic acid, we assumed that the relations might be indirectly resulted from their positive correlations with Pediococcus, Weissella, and Pichia.

\section{Conclusion}

In the current study, endogenous factors and bacterial and fungal communities in FGs during stacking fermentation for the production of Moutai-flavored liquor were comprehensively investigated. In general, changes in community diversity in both domains showed declining trends, and bacterial diversities had higher levels than fungal diversities during fermentation. The results of the community composition and EdgeR analyses generated 12 key bacteria and 12 key fungi, which had the major contribution to the variation of community succession. In addition, edgeR analysis further elucidated that the early and the middle fermentative periods were important for FGs to the structure of the key composition and that the functional populations accumulated mainly in the later periods. The endogenous factors showed different variation trends, and their potential associated key microbiota were also revealed by PLS-DA modeling. In conclusion, this study enhances the understanding of the dynamic changes in microbial communities and key microbes during stacking fermentation. The interactions among key microbiota and endogenous factors were discussed and considered, and theoretical support for optimizing microbial structures through the regulation of endogenous factors and improving fermentative quality was provided. 


\section{Acknowledgements}

This work was supported by grants from Science and Technology Foundation of Guizhou province (No. Qiankehe Jichu [2020]1Y076, Qiankehe Jichu [2019]1295, Qiankehe Jichu [2020]1Y144 and Qiankehe ZK [2021] General 093), Science and Technology Foundation of Guizhou Education Department (No. Qianjiaohe KY zi[2020]035 and Qianjiaohe KY zi [2018]003), and Foundation of 2019 Scientific research project of Moutai Institute [No. myk2019003].

\section{References}

Anderson, M. J. (2001). A new method for non-parametric multivariate analysis of variance. Austral Ecology, 26(1), 32-46.

Bai, D. M., Ban, R., Zhao, X. M., \& Hu, Z. D. (2000). Determination of lactic acid in fermentation broth of Rhizopus oryzae by reversedphase high performance liquid chromatography (RP-HPLC). Se Pu, 18(6), 527-528. PMid:12541741.

Beaumont, M. (2002). Flavouring composition prepared by fermentation with Bacillus spp. International Journal of Food Microbiology, 75(3), 189-196. http://dx.doi.org/10.1016/S0168-1605(01)00706-1. PMid:12036142.

Caporaso, J. G., Kuczynski, J., Stombaugh, J., Bittinger, K., Bushman, F. D., Costello, E. K., Fierer, N., Peña, A. G., Goodrich, J. K., Gordon, J. I., Huttley, G. A., Kelley, S. T., Knights, D., Koenig, J. E., Ley, R. E., Lozupone, C. A., McDonald, D., Muegge, B. D., Pirrung, M., Reeder, J., Sevinsky, J. R., Turnbaugh, P. J., Walters, W. A., Widmann, J., Yatsunenko, T., Zaneveld, J., \& Knight, R. (2010). QIIME allows analysis of high-throughput community sequencing data. Nature Methods, 7(5), 335-336. http://dx.doi.org/10.1038/nmeth.f.303. PMid:20383131.

Caporaso, J. G., Lauber, C. L., Walters, W. A., Berg-Lyons, D., Huntley, J., Fierer, N., Owens, S. M., Betley, J., Fraser, L., Bauer, M., Gormley, N., Gilbert, J. A., Smith, G., \& Knight, R. (2012). Ultra-highthroughput microbial community analysis on the Illumina HiSeq and MiSeq platforms. The ISME Journal, 6(8), 1621-1624. http:// dx.doi.org/10.1038/ismej.2012.8. PMid:22402401.

Caporaso, J. G., Lauber, C. L., Walters, W. A., Berg-Lyons, D., Lozupone, C. A., Turnbaugh, P. J., Fierer, N., \& Knight, R. (2011). Global patterns of $16 \mathrm{~S}$ rRNA diversity at a depth of millions of sequences per sample. Proceedings of the National Academy of Sciences of the United States of America, 108(1, Suppl. 1), 4516-4522. http://dx.doi. org/10.1073/pnas.1000080107. PMid:20534432.

Cheng, C. Y., Liu, X. F., Yuan, Y. X., Yan, Z. Y., Liao, Y. Z., \& Fu, S. (2014). Bacterial community structure in distiller's yeast and accumulated fermented grains of Maotai-flavor liquor. Chinese Journal of Applied and Environmental Biology, 20(5), 825-831.

Clarke, K. R. (1993). Non-parametric multivariate analyses of changes in community structure. Australian Journal of Ecology, 18(1), 117-143. http://dx.doi.org/10.1111/j.1442-9993.1993.tb00438.x.

Edgar, R. C. (2010). Search and clustering orders of magnitude faster than BLAST. Bioinformatics (Oxford, England), 26(19), 2460-2461. http://dx.doi.org/10.1093/bioinformatics/btq461. PMid:20709691.

Edgar, R. C., Haas, B. J., Clemente, J. C., Quince, C., \& Knight, R. (2011). UCHIME improves sensitivity and speed of chimera detection. Bioinformatics, 27(16), 2194-2200. http://dx.doi.org/10.1093/ bioinformatics/btr381. PMid:21700674.

Fadrosh, D. W., Ma, B., Gajer, P., Sengamalay, N., Ott, S., Brotman, R. M., \& Ravel, J. (2014). An improved dual-indexing approach for multiplexed 16S rRNA gene sequencing on the Illumina MiSeq platform. Microbiome, 2(1), 6. http://dx.doi.org/10.1186/2049-26182-6. PMid:24558975.

Fierer, N., Hamady, M., Lauber, C. L., \& Knight, R. (2008). The influence of sex, handedness, and washing on the diversity of hand surface bacteria. Proceedings of the National Academy of Sciences of the United States of America USA, 105(46), 17994-17999. http://dx.doi. org/10.1073/pnas.0807920105. PMid:19004758.

Huang, Y. L., Huang, Y. G., Hu, J. F., Hu, F., \& Zhong, F. D. (2017). Microbial diversity of the second rounds liquid of Moutai-flavor Baijiu during fermentation process. China Brewing, 36, 30-35. [In Chinese]

Kanehisa, M., Goto, S., Kawashima, S., Okuno, Y., \& Hattori, M. (2004). The KEGG resource for deciphering the genome. Nucleic Acids Research, 32(Database issue), D277-D280. http://dx.doi.org/10.1093/ nar/gkh063. PMid:14681412.

Karlsson, I., Friberg, H., Steinberg, C., \& Persson, P. (2014). Fungicide effects on fungal community composition in the wheat phyllosphere. PLoS One, 9(11), e111786. http://dx.doi.org/10.1371/journal. pone.0111786. PMid:25369054.

Lv, X. B., Wu, Y. L., Hao, F., Zeng, X. L., Zhang, Q. L., Chen, L. Q., Yuan, J., Luo, R. Y., Yang, F., Wang, H. Y., Wang, L., Wei, H. T., Han, P. J., \& Bai, F. Y. (2019). Analysis of microbial diversity in the 1st and 2nd fermentation Cycle of Moutaixiang baijiu based on high throughput sequencing technology. Liqour-making Science and Technology, 3, 52-64. In Chinese.

McMurdie, P. J., \& Holmes, S. (2014). Waste not, want not: why rarefying microbiome data is inadmissible. PLoS Computational Biology, 10(4), e1003531. http://dx.doi.org/10.1371/journal.pcbi.1003531. PMid:24699258.

Mylona, A. E., Del Fresno, M., Palomero, F., Loira, I., Bañuelos, M. A., Morata, A., Calderón, F., Benito, S., \& Suárez-Lepe, J. A. (2016). Use of Schizosaccharomyces strains for wine fermentation: effect on the wine composition and food safety. International Journal of Food Microbiology, 232, 63-72. http://dx.doi.org/10.1016/j. ijfoodmicro.2016.05.023. PMid:27261767.

Pagaling, E., Yang, K., \& Yan, T. (2014). Pyrosequencing reveals correlations between extremely acidophilic bacterial communities with hydrogen sulphide concentrations, $\mathrm{pH}$ and inert polymer coatings at concrete sewer crown surfaces. Journal of Applied Microbiology, 117(1), 50-64. http://dx.doi.org/10.1111/jam.12491. PMid:24606006.

Robinson, M. D., McCarthy, D. J., \& Smyth, G. K. (2010). edgeR: a bioconductor package for differential expression analysis of digital gene expression data. Bioinformatics, 26(1), 139-140. http://dx.doi. org/10.1093/bioinformatics/btp616. PMid:19910308.

Rohart, F., Gautier, B., Singh, A., \& Lê Cao, K.-A. (2017). mixOmics: An $\mathrm{R}$ package for 'omics feature selection and multiple data integration. PLoS Computational Biology, 13(11), e1005752. http://dx.doi. org/10.1371/journal.pcbi.1005752. PMid:29099853.

Song, Z. W., Du, H., Zhang, Y., \& Xu, Y. (2017). Unraveling core functional microbiota in traditional solid-state fermentation by high-throughput amplicons and metatranscriptomics sequencing. Frontiers in Microbiology, 8, 1294. http://dx.doi.org/10.3389/ fmicb.2017.01294. PMid:28769888.

Sun, L. L., Li, L. L., Hu, P., Tian, Y., Ma, Y. Y., \& Yuan, Z. S. (2019). Analysis of fungal diversity in Maotai-flavor baijiu during the fourth round fermentation. China Brewing, 38, 24-30. In Chinese.

Wang, C., Shi, D., \& Gong, L. (2008). Microorganisms in Daqu: a starter culture of Chinese Maotai-flavor liquor. World Journal of Microbiology \& Biotechnology, 24(10), 2183-2190. http://dx.doi. org/10.1007/s11274-008-9728-0. 
Wang, M. Y., Yang, J. G., Zhao, Q. S., Zhang, K. Z., \& Su, C. (2019). Research progress on flavor compounds and microorganisms of Maotai flavor baijiu. Journal of Food Science, 84(1), 6-18. http:// dx.doi.org/10.1111/1750-3841.14409. PMid:30548499.

Wang, X. S., Du, H., Zhang, Y., \& Xu, Y. (2018). Environmental microbiota drives microbial succession and metabolic profiles during Chinese liquor fermentation. Applied and Environmental Microbiology, 84(4), e02369-17. http://dx.doi.org/10.1128/AEM.02369-17. PMid:29196296.

Wu, Q., Chen, B., \& Xu, Y. (2015). Regulating yeast flavor metabolism by controlling saccharification reaction rate in simultaneous saccharification and fermentation of Chinese Maotai-flavor liquor. International Journal of Food Microbiology, 200, 39-46. http://dx.doi. org/10.1016/j.ijfoodmicro.2015.01.012. PMid:25676241.

Wu, Q., Chen, L. Q., \& Xu, Y. (2013). Yeast community associated with the solid state fermentation of traditional Chinese Maotai-flavor liquor. International Journal of Food Microbiology, 166(2), 323-330. http://dx.doi.org/10.1016/j.ijfoodmicro.2013.07.003. PMid:23978339.

Wu, Q., Xu, Y., \& Chen, L. (2012). Diversity of yeast species during fermentative process contributing to Chinese Maotai-flavor liquor making. Letters in Applied Microbiology, 55(4), 301-307. http:// dx.doi.org/10.1111/j.1472-765X.2012.03294.x. PMid:22862564.

Wu, Z.-Y., Lei, X.-J., Zhu, D.-W., \& Luo, A.-M. (2016). Investigating the variation of volatile compound composition in Maotai flavour liquor during its multiple fermentation steps using statistical methods.
Food Technology and Biotechnology, 54(2), 243-249. http://dx.doi. org/10.17113/ftb.54.02.16.4582. PMid:27904415.

Xiong, Z. (1994). The manufactures of moutai-flavor liquor. Beijing: Light Industry Publishing House of China.

Xiu, L., Kunliang, G., \& Hongxun, Z. (2012). Determination of microbial diversity in Daqu, a fermentation starter culture of Maotai liquor, using nested PCR-denaturing gradient gel electrophoresis. World Journal of Microbiology \& Biotechnology, 28(6), 2375-2381. http:// dx.doi.org/10.1007/s11274-012-1045-y. PMid:22806111.

Xu, Y., \& Ji, K. (2012). Moutai (Maotai): production and sensory properties. In J. Piggott (Ed.), Alcoholic beverages: sensory evaluation and consumer research (pp. 315-330). Cambridge: Woodhead Publishing. http://dx.doi.org/10.1533/9780857095176.3.315.

Yang, F., Chen, L. Q., Liu, Y. F., Li, J. H., Wang, L., \& Chen, J. (2019). Identification of microorganisms producing lactic acid during solidstate fermentation of Maotai flavour liquor. Journal of the Institute of Brewing, 125(1), 171-177. http://dx.doi.org/10.1002/jib.537.

Yang, F., Lin, L., Wang, H. Y., Wang, L., Yang, D. Y., Lu, Y. H., \& Ji, K. L. (2011). Comparative analysis of the metabolites of three Bacillus strains in Maotai Daqu starter. Niangjiu Ke-Ji, 32, 42-46. In Chinese.

Ying, C. C., Liu, X. F., Yuan, Y. X., Yan, Z. Y., Liao, Y. Z., Fu, S. (2014). Bacterial community structure in distiller's yeast and accumulated fermented grains of Maotai-flavor liquor. Chinese Journal of Applied and Environmental Biology, 20(5), 825-831. In Chinese. 


\section{Supplementary Material}

Supplementary material accompanies this paper.

Table S1. Diversity measurements of the two domains' communities and contents of endogenous factors in fermented grains at different fermentation periods.

Table S2. Bacterial and Fungal genera that were significantly differentially abundant during fermentation as compared with P1 (Excel database). Figure S1. Sampling locations of FGs in stacking fermentation. In the stack, sampling locations a, b, c, d and e correspond to the horizontal depth $30 \mathrm{~cm}, 60 \mathrm{~cm}, 90 \mathrm{~cm}, 120 \mathrm{~cm}$ and $150 \mathrm{~cm}$, respectively.

This material is available as part of the online article from https://www.scielo.br/j/cta 